\title{
Extreme-ultraviolet pump-probe studies of one-femtosecond-scale electron dynamics
}

\author{
P. Tzallas $^{1 \star}$, E. Skantzakis ${ }^{1,2}$, L. A. A. Nikolopoulos ${ }^{3}$, G. D. Tsakiris ${ }^{4}$ and D. Charalambidis ${ }^{1,2}$
}

\begin{abstract}
Ultrafast-dynamics studies and femtosecond-pulse metrology both rely on the nonlinear processes induced solely by an incident light pulse. Extending these approaches to the extreme-ultraviolet (XUV) spectral region would open up a new route to attosecond-scale dynamics. However, this has been hindered by the limited intensities available in coherent XUV continua. In the present work, we realized conditions at which simultaneous ejection of two bound electrons by two-XUVphoton absorption becomes more efficient than their removal one-by-one. In this regime we have succeeded in tracing atomic coherences evolving at the 1-fs scale with simultaneous determination of the average XUV-pulse duration. The rich and dense structure of the autoionizing manifold demonstrates the applicability of the approach to complex systems. This initiates the era of XUV-pump-XUV-probe experiments at the boundary between femto- and attosecond scales.
\end{abstract}

A large variety of ultrafast phenomena, including electronic motion in atoms, molecules, condensed matter and plasmas, dynamic electron-electron correlations, charge migration, ultrafast dissociation and reaction processes, occur on the few-femtosecond to attosecond temporal scale. Attosecond (as) pulses ${ }^{1}$ provide access to these temporal regimes in different states of matter ${ }^{2-6}$. Nonlinear (NL) XUV processes constitute the ideal tool for the study of such dynamics. Attosecond pulse trains ${ }^{7-9}$ have reached intensities sufficient to induce two-XUV-photon processes ${ }^{10-14}$. However, isolated attosecond pulses, requisite for XUV-pump-XUV-probe experiments, have not yet attained the required parameters for an observable two-XUV-photon process. As a consequence, attosecond pulse metrology and time-domain applications have been widely based on infrared (IR)-XUV cross-correlation approaches, which entail assumptions for the analysis ${ }^{15}$.

The present work succeeds for the first time in observing two-XUV-photon processes induced by energetic XUV continua, in part temporally confined in isolated pulses with durations on the order of $1 \mathrm{fs}$. These processes are in turn exploited in XUVpump-XUV-probe ultrafast evolving atomic coherences, as well as in determining the duration of the XUV bursts. A structured part of the single continuum of the xenon atom is excited by the first pulse, forming an electronic wave packet that undergoes rapid and complex motion before it decays. This evolution can be traced, thanks to the XUV parameters reached, at which a second pulse ejects a second electron before the first one leaves the atom carrying with it all the information on the temporal evolution of the system (coherence decay). Unconventionally, the two electrons leave the atom together and, thus, the doubly ionized Xe yield as a function of the delay between the two pulses carries the fingerprint of the wave packet motion and the XUV pulse duration. As the pulse duration and the decay time of the wave packet are very different, pulse duration and wave packet dynamics are imprinted in different regions of the measured trace.

The intense XUV radiation is generated by frequency upconversion of many-cycle high-peak-power laser fields interacting with an atomic target. To obtain the XUV continua, the technique of Interferometric Polarization Gating (IPG; refs 16,17) is applied. As the laser used is not carrier-envelope phase(CEP)-stabilized, only a fraction of the laser shots generate pure XUV continuum spectra and thus, single pulses ${ }^{18}$. Pulses with CEP far from $\pi / 2$ generate modulated spectra ${ }^{16,18}$, resulting in waveforms with a double-peak structure. The discussion to follow will be confined to pure continua. Possible modifications of the results arising from the shot-to-shot CEP variation will be further considered separately.

The experimental set-up used is shown in Fig. 1a. Ti:Sapph pulses of length $38 \mathrm{fs}$ enter an IPG device (see Supplementary Information). The ellipticity modulated output pulses interact with a xenon gas jet to produce the XUV radiation. The radiation transmitted through a Sn filter spans the spectral range $50-80 \mathrm{~nm}$ (Fig. 1b). The XUV radiation is focused into a second gas jet (He or $\mathrm{Xe}$ ) by a bisected spherical mirror acting also as a wavefront splitter. Translation of one mirror half produces two mutually delayed pulses with variable delay.

Two-XUV-photon processes were observed in two different experiments. In the first, two-photon ionization of $\mathrm{He}$ was verified by the dependence of the ion signal on the XUV intensity (see Supplementary Information). In the second, doubly ionized xenon was observed in the measured mass ion spectra (Fig. 1c). For the given XUV spectral width, $\mathrm{Xe}^{2+}$ can only be produced through multi-XUV-photon absorption (Fig. 2). The most dominant possible channels are: (1) two-photon direct double ionization (TPDDI) in which single XUV photon absorption is in the vicinity of a manifold of doubly excited $5 s^{2} 5 p^{4}\left[{ }^{3} P,{ }^{1} D,{ }^{1} S\right] m l m^{\prime} l^{\prime}$ and inner-shell excited $5 s 5 p^{6}\left({ }^{2} S_{1 / 2}\right) n p\left[{ }^{1} P_{1}\right]$ (Auger) autoionizing states (AIS), from where absorption of a second XUV-photon ejects two electrons that share the excess energy and (2) singleXUV-photon ionization of Xe, followed by a single-XUV-photon ionization of the two fine structure levels of the $\mathrm{Xe}^{+}$ground state by the high energy (above 21 or $19.7 \mathrm{eV}$ for each level) part of the continuum spectrum distribution that exceeds the double ionization threshold (sequential double ionization (SDI)). Further, less probable sequential channels through excited ionic states are discussed in the Supplementary Information. For pulse durations $\tau_{\mathrm{XUV}}$ of tens of $\mathrm{fs}$ the sequential two-XUV-photon ionization is the dominant process. As pulse duration decreases, the direct process increases in relative strength. The reason for this relates to the different dependence on the pulse duration of the direct $\left(\propto \tau_{\mathrm{XUV}}\right)$ and sequential $\left(\propto \tau_{\mathrm{XUV}}^{2}\right)$ ionization process ${ }^{19}$. At the $1 \mathrm{fs}$

\footnotetext{
${ }^{1}$ Foundation for Research and Technology-Hellas, Institute of Electronic Structure and Laser, PO Box 1527, GR-711 10 Heraklion, Crete, Greece, ${ }^{2}$ Department of Physics, University of Crete, PO Box 2208, GR71003 Heraklion, Crete, Greece, ${ }^{3}$ School of Physical Sciences, Dublin City University, Glasnevin, Dublin 9, Ireland, ${ }^{4}$ Max-Planck-Institut für Quantenoptik, Hans-Kopfermann-Str. 1, D-85748 Garching, Germany. *e-mail: ptzallas@iesl.forth.gr.
} 


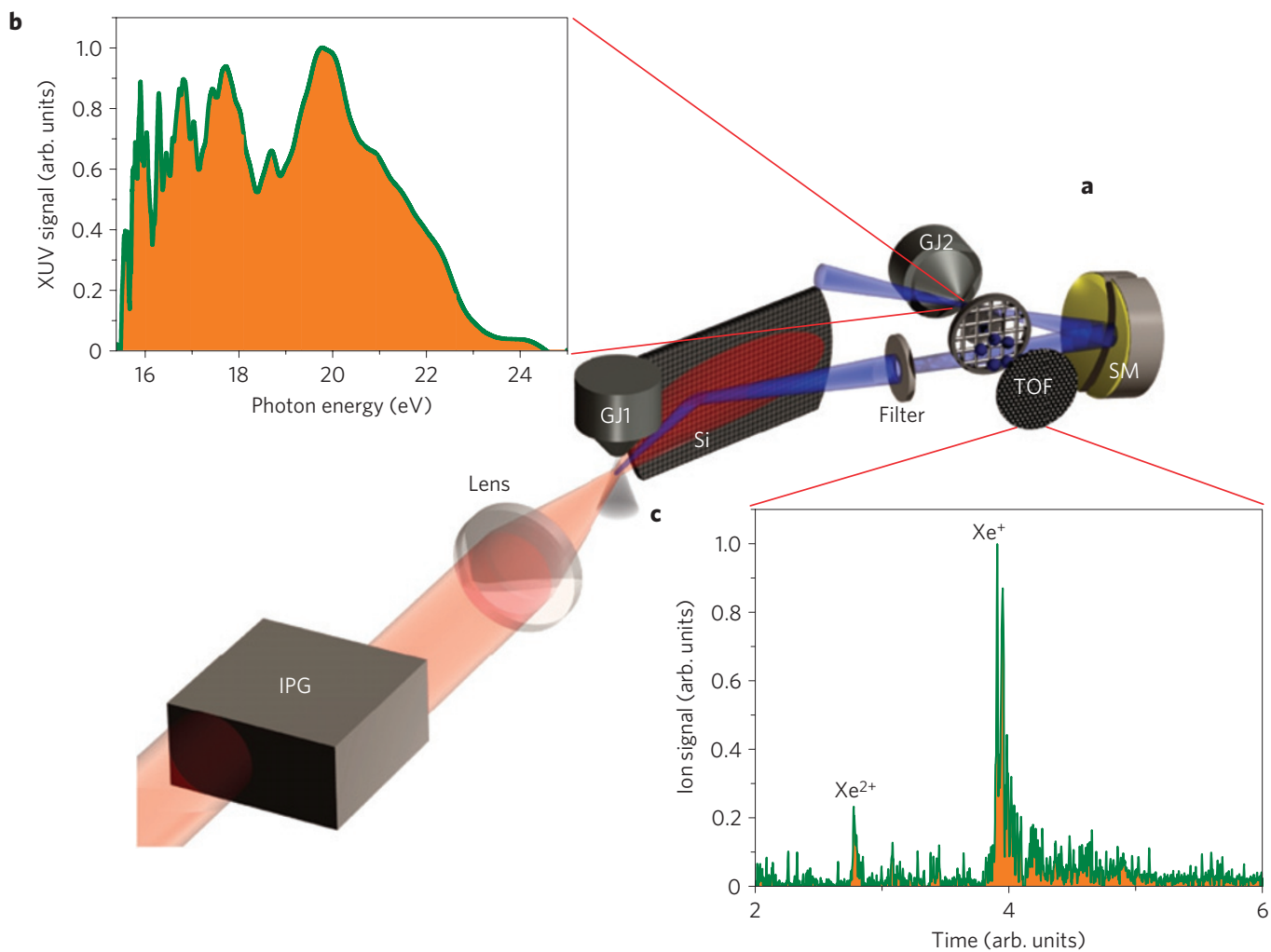

Figure 1 | Schematic diagram showing the XUV-pump-XUV-probe apparatus. a, Apparatus for the XUV-pump-XUV-probe studies and the temporal characterization of an isolated XUV burst (see Supplementary Information). The XUV-broadband coherent continuum radiation is generated in the Xe gas jet (GJ1) by means of an IPG device. The XUV radiation, after the reflection on a Si plate, passes through an aperture and a thin Sn filter, selecting the desired spectral range. Subsequently, it is focused into the target gas jet (GJ2) by means of a gold split-spherical mirror XUV autocorrelator. $\mathbf{b}$, The spectrum (average of 300 pulses) of the radiation used in the experiment. In the interaction region, this radiation can support isolated pulses with durations as short as $\approx 420$ as and intensities exceeding $10^{14} \mathrm{~W} \mathrm{~cm}^{-2}$. c. Focusing this radiation in a xenon gas target, the charge states of $\mathrm{Xe}^{+}$and $X \mathrm{e}^{2+}$ have been observed in the TOF spectrum. The green lines are the border lines of the orange filled areas.

pulse duration level, the direct process becomes the strongest double-ionization channel; verified experimentally in this work. Other correlation effects occurring in the strong-field tunnelling regime, such as those presented in ref. 20, seem to be less relevant to our case, in which interactions are perturbative and in the multi-photon regime.

Using a sequence of two XUV pulses, and varying the delay $\Delta \tau$ between them, we are able to: (1) measure their duration by means of a second order intensity volume autocorrelation (second order IVAC) from the part of the trace in which the two pulses are overlapping and (2) induce, control and probe a fast-evolving coherence in the structured continuum, by evaluating the part of the trace where the two pulses are not overlapping. Here, the first pulse (P1) induces the coherence, pumping a coherent superposition of the AIS manifold and ionic states. It also partially doubly ionizes the atom, ejecting part of the formed electron wave packet. The second pulse (P2): (1) excites a replica of the wave packet, which interferes with the evolved first one. Thus it controls the excitation by means of constructive or destructive interference occurring at different frequencies. (2) Partially ionizes the wave packet it excites and probes the evolution of the first excited wave packet. The path involving excitation by $\mathrm{P} 1$ and double ionization by $\mathrm{P} 2$ is, to our knowledge, the first successful experimental implementation of an XUV-pump-XUV-probe sequence.

The measured trace is shown in Fig. 3a. The pronounced maximum around $\Delta \tau=0$ is the second order IVAC trace. This is because contributions to this part are predominantly due to the TPDDI channel, (1) because of the shortness of the pulse and (2) because only a small portion of the spectrum contributes

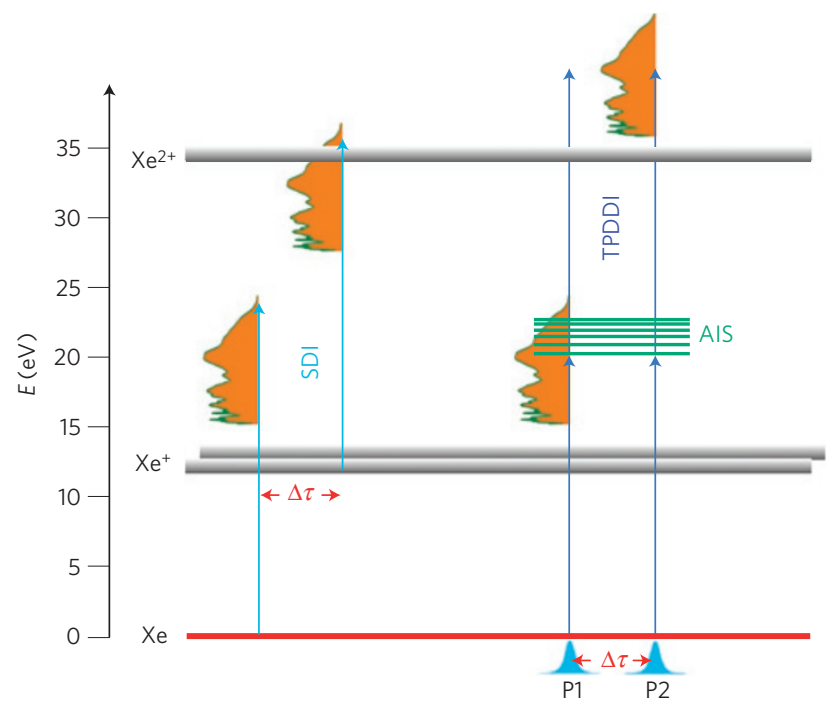

Figure 2 | Nonlinear process used for the temporal characterization of isolated XUV pulses and the probing of ultrafast evolving atomic coherences. Schematic diagram of the multi-XUV-photon ionization process induced in xenon by the interaction with the sequence of two mutually delayed intense broadband XUV pulses. The two non-negligible competing channels leading to double ionization are: (1) two-photon direct double ionization (TPDDI), in part through the AIS manifold, and (2) two-photon sequential double ionization (SDI). At the $1 \mathrm{fs}$ or even sub-fs pulse duration level the direct process dominates. 
a

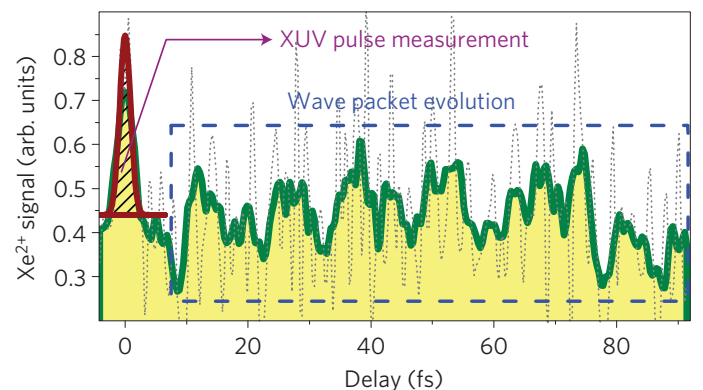

b

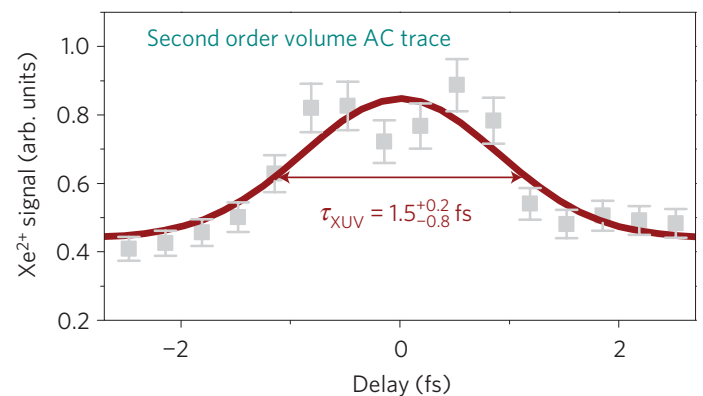

c

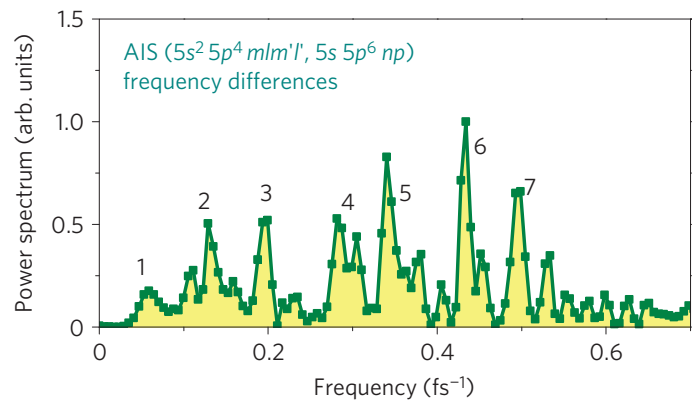

Figure 3 | XUV-pump-XUV-probe measurement of one

femtosecond-scale electron dynamics. a, Second order autocorrelation trace retrieved by recording the dependence of the $\mathrm{Xe}^{2+}$ signal on the delay between the XUV pulses. The grey dashed line corresponds to the raw data. To make the trend of the trace clearer, the moving averages of the raw data are taken over nine delay points (green-yellow-filled area). Two domains are clearly distinguishable in the trace. The interval around zero delay ( $-3 \mathrm{fs}$ to $6 \mathrm{fs}$ ) serves for the XUV pulse characterization and the trace at longer delays ( $6 \mathrm{fs}$ to $92 \mathrm{fs}$ ) traces the evolution of the induced atomic coherences. The purple-line-shaded curve is the Gaussian fit on the raw data in the area, where the two XUV parts are temporally overlapped. b. An expanded area of the trace in $\mathbf{a}$. The Gaussian fit on the raw data yields $\tau_{X U V}=1.5_{-0.8}^{+0.2} \mathrm{fs}$. Note that the lower position of the two points left and right of zero delay is within the fluctuations of the measured points and thus the 'double-peak structure' that appears is not a real structure. The error bars are statistical errors equal to one standard deviation. c, FT of the raw data of trace $\mathbf{a}$ for delays $>6$ fs reveals the frequency differences of the excited states. The frequency resolution, resulting from the maximum delay between the two mutually delayed pulses, is $\sim 0.03 \mathrm{fs}^{-1}$. The assignment of the numbered peaks can be found in the Supplementary Information.

to the sequential channel. A Gaussian distribution fit (Fig. 3b) results in a pulse duration of $1.5 \mathrm{fs}$. It is well known ${ }^{16,18}$ that because of the shot to shot CEP variation, the spectrum varies from a pure to a modulated continuum, which, in the time domain, translates to a variation between a clean single pulse and pulses with a double-maxima structure. Thus, the measured duration of $1.5 \mathrm{fs}$ is the average of the duration of single pulses, possibly of sub-fs duration and twin maxima pulses with a peak separation of half of the laser period ( $1.33 \mathrm{fs}$ ). Consequently, the measured duration is $\tau_{\mathrm{XUV}}=1.5_{-0.8}^{+0.2} \mathrm{fs}$ ( $420 \mathrm{as}$ being the Fourier Transform Limited (FTL) duration). This duration further verifies the dominance of the TPDDI channel ${ }^{19}$. A SDI trace would be the cycle-average of the square of the first order autocorrelation of the field ${ }^{21}$, and thus would consist of a main and two lower side peaks, each having a width equal to the width of the FT of the XUV spectrum (420 as). The much larger measured width, together with the high peak to background ratio (see Supplementary Information), confirms that the two electrons are ejected 'together' before the system finds the time to first decay to $\mathrm{Xe}^{+}+\mathrm{e}^{-}$.

The beating signal at delays $>5 \mathrm{fs}$ results from the XUVpump-XUV-probe process of the atomic coherence. At the temporal resolution of the experiment, high-frequency Ramsey fringes corresponding to the excitation frequencies of each AIS are averaged out and the low-frequency components of the evolution of the coherent superposition dominate the trace ${ }^{22}$. Contributions from modulations in the excitation process due to the two interfering wave packets are not to be excluded ${ }^{23,24}$. Fourier transform (FT) of the traces reveals frequency differences of the excited states (Fig. 3c). An assignment of the FT spectrum peaks is given in the Supplementary Information. Most of the peak positions coincide, within the error, with those measured in ref. 5, where the dynamics were observed at the autoionization process. The double-peak structure of some XUV pulses, due to the variation of the CEP, modifies the exciting amplitude distribution and appears in the trace components shifted by half the laser period, which only reduces the fringe contrast in the averaged trace.

In a similar study, involving only bound states and infrared fs pulses, a theoretical description is given in the weak field approximation $^{22}$. In the present conditions this approximation might not be valid, as ground state depletion and higher order couplings cannot be excluded. As calculation of the relevant xenon atomic structure is not tractable, we present $a b$ initio timedependent perturbative calculations of the appropriate order in helium (see Supplementary Information).

In summary, the present work establishes the era of XUVpump-XUV-probe experiments at the $1 \mathrm{fs}$ temporal scale, along with isolated pulse metrology at this temporal scale, based solely on NL-XUV processes. The rich and dense structure investigated in this part of the spectrum indicates applicability of the approach to complex systems. In this sense the present experiment opens up a new chapter in time-domain studies of realistic complex systems at ultra-high temporal resolution.

\section{Received 1 March 2011; accepted 27 May 2011; published online} 3 July 2011

\section{References}

1. Krausz, F. \& Ivanov, M. Attosecond Physics. Rev. Mod. Phys. 81, 163-234 (2009).

2. Remetter, T. et al. Attosecond electron wave packet interferometry. Nature Phys. 2, 323-326 (2006).

3. Cavalieri, A. L. et al. Attosecond spectroscopy in condensed matter. Nature 449, 1029-1032 (2007).

4. Sansone, G. et al. Electron localization following attosecond molecular photoionization. Nature 465, 763-766 (2010).

5. Skantzakis, E. et al. Tracking autoionizing-wave-packet dynamics at the 1-fs temporal scale. Phys. Rev. Lett. 105, 043902 (2010).

6. Goulielmakis, E. et al. Real-time observation of valence electron motion. Nature 466, 739-743 (2010).

7. Tzallas, P., Charalambidis, D., Papadogiannis, N. A, Witte, K. \& Tsakiris, G. D. Direct observation of attosecond light bunching. Nature 426, 267-271 (2003).

8. Nabekawa, Y. et al. Conclusive evidence of an attosecond pulse train observed with the mode-resolved autocorrelation technique. Phys. Rev. Lett. 96, 083901 (2005).

9. Nomura, Y. et al. Attosecond phase locking of harmonics emitted from laser-produced plasmas. Nature Phys. 5, 124-128 (2009).

10. Xenakis, D., Faucher, O., Charalambidis, D. \& Fotakis, C. Observation of two-XUV-photon ionization using harmonic generation from a short, intense laser pulse. J. Phys. B 29, L457-L463 (1996). 
11. Papadogiannis, N. A. et al. Two-photon ionization of He through a superposition of higher harmonics. Phys. Rev. Lett. 90, 133902 (2003).

12. Sekikawa, T., Ohno, T., Yamazaki, T., Nabekawa, Y. \& Watanabe, S. Pulse compression of a high-order harmonic by compensating the atomic dipole phase. Phys. Rev. Lett. 83, 2564-2567 (1999).

13. Sekikawa, T., Katsura, T., Miura, S. \& Watanabe, S. Measurement of the intensity-dependent atomic dipole phase of a high harmonic by frequency-resolved optical gating. Phys. Rev. Lett. 88, 193902 (2002).

14. Nabekawa, Y., Hasegawa, H., Takahashi, E. J. \& Midorikawa, K. Production of doubly charged helium ions by two-photon absorption of an intense sub-10-fs soft X-ray pulse at $42 \mathrm{eV}$ photon energy. Phys. Rev. Lett. 94, 043001 (2005).

15. Kruse, J. E. et al. Inconsistencies between two attosecond pulse metrology methods: A comparative study. Phys. Rev. A 82, 021402(R) (2010).

16. Tzallas, P. et al. Generation of intense continuum extreme-ultraviolet radiation by many cycle laser fields. Nature Phys. 3, 846-850 (2007).

17. Skantzakis, E., Tzallas, P., Kruse, J., Kalpouzos, C. \& Charalambidis, D. Coherent continuum extreme ultraviolet radiation in the sub 100-nJ range generated by a high-power many-cycle laser field. Opt. Lett. 34, 1732-1734 (2009).

18. Tzallas, P., Skantzakis, E. \& Charalambidis, D. Measuring the absolute carrier-envelope phase of many-cycle laser fields. Phys. Rev. A 82, 061401(R) (2010).

19. Lambropoulos, P., Nikolopoulos, L. A. A., Makris, M. G. \& Mihelič, A. Direct versus sequential double ionization in atomic systems. Phys. Rev. A 78, 055402 (2008).

20. Pfeiffer, A. N., Cirelli, C., Smolarski, M., Dörner, R. \& Keller, U. Timing the release in sequential double ionization. Nature Phys. 7, 428-433 (2011).

21. Nakajima, T. \& Nikolopoulos, L. A. A. Use of helium double ionization for autocorrelation of an XUV pulse. Phys. Rev. A 66, 041402(R) (2002).
22. Blanchet, V., Bouchène, M. A. \& Girard, B. Temporal coherent control in the photoionization of $\mathrm{Cs}_{2}$ : Theory and experiment. J. Chem. Phys. 108 4862-4876 (1998).

23. Campbell, M. B., Bensky, T. J. \& Jones, R. R. Observation of oscillations between degenerate bound-state configurations in rapidly autoionizing two-electron atoms. Phys. Rev. A 57, 4616-4620 (1998).

24. Jones, R. R., Raman, C. S., Schumacher, D. W. \& Bucksbaum, P. H. Ramsey interference in strongly driven Rydberg systems. Phys. Rev. Lett. 71, 2575-2578 (1993).

\section{Acknowledgements}

This work is supported in part by the European Commission programs ULF, ALADIN (GA-228334), ATTOFEL (GA-238362), FASTQUAST (PITN-GA-2008-214962), ELI-PP (GA-212105) and FLUX program (PIAPP-GA-2008-218053) of the 7th FP. L.A.A.N acknowledges the SFI Stokes Lectureship program, COST CM0702 action and the SFI/HEA ICHEC.

\section{Author contributions}

P.T. was the main contributor in all aspects of this work; E.S. contributed to the experimental runs and data analysis; L.A.A.N. performed the theoretical calculations; G.D.T. contributed to the design of the experiment and the preparation of the manuscript; D.C. supervised the project.

\section{Additional information}

The authors declare no competing financial interests. Supplementary information accompanies this paper on www.nature.com/naturephysics. Reprints and permissions information is available online at http://www.nature.com/reprints. Correspondence and requests for materials should be addressed to P.T. 